\title{
ESTUDO RETROSPECTIVO DE 71 CASOS DE PROTRUSÃO DA GLÂNDULA DA TERCEIRA PÁLPEBRA (2009-2013)
}

\author{
Natalie Bertelis Merlini ${ }^{1}$ \\ Úrsula Chaves Guberman² \\ Micaella Gordon Gandolfi ${ }^{3}$ \\ Vivian Lima de Souza ${ }^{2}$ \\ Natália Rodrigues Rodas ${ }^{2}$ \\ José Joaquim Titton Ranzani ${ }^{4}$ \\ Cláudia Valéria Seullner Brandão ${ }^{5}$
}

MERLINI, N. B.; GUBERMAN, U. C.; GANDOLFI, M. G.; SOUZA, V. L. de; RODAS, N. R.; RANZANI, J. J. T.; BRANDÃO, C. V. S. Estudo retrospectivo de 71 casos de protrusão da glândula da terceira pálpebra (2009-2013). Arq. Ciênc. Vet. Zool. UNIPAR, Umuarama, v. 17, n. 3, p. 177-180, jul./set. 2014.

\begin{abstract}
RESUMO: Objetivou-se com esse estudo avaliar a incidência de protrusão da glândula da terceira pálpebra e correlacionar as características de raça e sexo com a lateralidade da alteração. Para isso, foram analisadas 3.151 fichas clínicas atendidas setor de oftalmologia veterinária da Universidade Estadual de São Paulo (UNESP) no período de 2009 a 2013, avaliando-se dados de incidência e lateralidade da afecção, além de idade, sexo e raça dos animais acometidos. Realizou-se a análise estatística de frequência e calculou-se o coeficiente de correlação de Spearman, considerando o nível de significância de 5\%. Foram diagnosticados, no total, 71 casos de protrusão da glândula da terceira pálpebra em cães, correspondendo a 2,43\% (71/2.916) da casuística de atendimento oftalmológico nesta espécie, porém não foi observado nenhum caso em gatos. Obteve-se uma maior frequência $(58 \%, 41 / 71)$ em cães com idade inferior a 12 meses, sendo as raças mais acometidas: Lhasa Apso (24\%, 17/71), Sem Raça Definida (20\%, 14/71) e Beagle (14\%, 10/71). Em 72\% (44/71) dos casos a afecção se manifestou unilateral e 55\% (39/71) dos animais eram fêmeas. Não houve correlação entre o sexo e a raça com a lateralidade da protrusão (p $>0,05)$. Com base no levantamento de dados realizados nesta instituição, conclui-se que a incidência da afecção é baixa em cães e rara em gatos, além de que não há influência do sexo e da raça com relação ao lado acometido pela alteração.
\end{abstract}

PALAVRAS-CHAVE: Cães. Cherry eyes. Gatos. Membrana nictitante. Prolapso.

\section{RETROSPECTIVE STUDY OF 71 CASES OF PROLAPSED THIRD EYELID GLAND (2009-2013)}

ABSTRACT: This paper aims to analyze the incidence of prolapsed third eyelid gland and correlate the breed and gender characteristics to the laterality of the alteration. In order to do this, three thousand one hundred and fifty-one (3151) medical records were analyzed at the Department of veterinary ophthalmology from the State University of São Paulo (UNESP) from 2009 to 2013, considering data such as the incidence and laterality of the disease, as well as age, gender and breed of affected animals. A frequency statistical analysis was performed and the Spearman correlation coefficient was calculated by taking the significance level of 5\%. The prolapse of the third eyelid gland was diagnosed in 71 cases in dogs, which corresponds to $2.43 \%(71 / 2916)$ of the cases cared for in the ophthalmological assistance in this specie; however, no cases were observed in cats. Dogs younger than 12 months of age presented a higher frequency, (58\%, 41/71). The breeds most affected were Lhasa Apso (24\%, 17/71); Mongrel (20\%, 14/71); and Beagle (14\%, 10/71). The prolapse was unilateral in 72\% (44/71) of the cases, and 55\% (39/71) of the animals were female. There was no significant relation of gender and breed with the laterality of the prolapsed gland $(\mathrm{p}>0.05)$. Therefore, it can be concluded that the incidence of the alteration is low in dogs and rare in cats, as well as no influence being related to gender and breed to the side affected by the alteration.

KEYWORDS: Dogs. Cherry eyes. Cats. Nictitating membrane. Prolapse.

\section{ANÁLISIS RETROSPECTIVO DE 71 CASOS DE PROLAPSOS DE GLÁNDULA DEL TERCER PÁRPADO} (2009-2013)

RESUMEN: El objetivo de esta investigación ha sido evaluar la incidencia del prolapso de la glándula del tercer parpado y correlacionar las características de raza y sexo con la lateralidad de la alteración. Para tanto fueron analizadas 3151 fichas clínicas atendidas en el sector de oftalmología veterinaria de la Universidade Estadual de São Paulo (UNESP) entre 2009 y 2013, considerando datos de incidencia y lateralidad de la afección, además de edad, sexo y raza de los animales enfermos. Se realizó el análisis estadístico de frecuencia y fue calculado el coeficiente de correlación de Spearman, considerando el nivel DOI: https://doi.org/10.25110/arqvet.v17i3.2014.4941

'Médica Veterinária, pós graduanda (Doutorado) em Biotecnologia Animal, Universidade Estadual Paulista “Júlio de Mesquita Filho", Campus de Botucatu. natalie_merlini@hotmail.com

${ }^{2}$ Médica Veterinária, pós graduanda (Mestrado) em Biotecnologia Animal, Universidade Estadual Paulista "Júlio de Mesquita Filho", Campus de Botucatu. ${ }^{3}$ Médica Veterinária Autônoma.

${ }^{4}$ Professor Dr. assistente. Universidade Estadual Paulista "Júlio de Mesquita Filho". Departamento de Cirurgia e Anestesiologia Veterinária. Distrito de Rubião Júnior, Botucatu-SP.

${ }^{5}$ Professor Dra. Adjunta. Universidade Estadual Paulista "Júlio de Mesquita Filho". Departamento de Cirurgia e Anestesiologia Veterinária. Distrito de Rubião Júnior, Botucatu-SP. 
de significancia de 5\%. Se ha diagnosticado 71 casos de prolapso de la glándula del tercer parpado en caninos, correspondiendo a 2,43\% (71/2916) de la casuística del atendimiento oftalmológico en esta especie, sin embargo no se ha observado ningún caso en gatos. Hubo una frecuencia más grande $(58 \%, 41 / 71)$ en perros con edad inferior a 12 meses, teniendo en cuenta que las razas que más presentaron este problema fueron Lhasa Apso (24\%, 17/71), Sin raza definida $(20 \%$, 14/71) y Beagle (14\%, 10/71). En 72\% (44/71) de los casos, la afección se manifestó unilateral y 55\% (39/71) de los animales eran hembras. No hubo correlación significativa entre el sexo y la raza con la lateralidad del prolapso ( $p>0,05)$. Con base en el alzamiento de datos realizados en esta institución, se ha concluido que la incidencia de la afección es baja en perros y rara en gatos, además de no haber influencia del sexo y de la raza con relación al lado tomado por la alteración.

PALABRAS CLAVE: Perros; Cherry eyes; Gatos; Membrana nictitante; Prolapso.

\section{Introdução}

A protrusão da glândula é a alteração mais comum da terceira pálpebra em cães (HENDRIX, 2007), e apesar de rara também é descrita em gatos (SCHOOFS, 1999; CHAHORY et al., 2004). Acomete principalmente animais jovens, sendo descritas como as principais raças acometidas: Beagle, Shih-Tzu, Mastin Napolitano (PEIXOTO; GALERA 2012), Lhasa Apso, Cocker Spaniel Americano, Bulldog Francês, Bulldog Inglês e Cane Corso (MAZZUCCHELLI et al., 2012). A etiologia desta afecção ainda não é bem definida, porém há suspeita de que seja decorrente de uma deformidade/frouxidão no retináculo, que é responsável por unir a glândula à periórbita (SLATTER, 2005; HENDRIX, 2007).

A glândula, quando protruída, fica exposta e visível no canto medial do olho (HENDRIX, 2007), sendo que essa exposição resulta em inflamação (SLATTER, 2005) e gera sinais clínicos como conjuntivite, secreção ocular (WOUK; SOUZA; FARIAS, 2009) e diminuição da produção lacrimal nas glândulas expostas cronicamente (DUGAN et al., 1992; CABRAL et al., 2008). Por meio da histopatologia, pode-se observar hiperplasia linfoide da terceira pálpebra (WOUK; SOUZA; FARIAS, 2009). O tratamento de eleição é o cirúrgico, sendo indicado reposicionamento da glândula protruída (CABRAL et al., 2008).

Objetivou-se com este estudo avaliar a incidência de protrusão da glândula da terceira pálpebra em cães e gatos, além de avaliar e correlacionar as características de raça e sexo com a lateralidade da afecção.

\section{Material e Métodos}

Foi realizado um levantamento retrospectivo dos casos de protrusão da glândula da terceira pálpebra em cães e gatosatendidos no setor de Oftalmologia Veterinária da Faculdade de Medicina Veterinária e Zootecnia na Universidade Estadual de São Paulo (UNESP), campus Botucatu. Foram analisadas, no total, 3.151 fichas clínicas dos casos oftálmicos, sendo 2.916 de cães e 235 de gatos, atendidos nos anos de 2009 a 2013.

Realizou-se a avaliação da incidência da afecção e a análise da lateralidade da afecção, além das variáveis de idade, sexo e raça dos pacientes acometidos pela protrusão da glândula da terceira pálpebra. Devido à imprecisão da data de nascimento dos animais, a faixa etária destes foi dividida em: filhotes (zero a um ano), adultos (entre um e sete anos) e idosos (mais de sete anos).

Os dados obtidos foram registrados, codificados e tabulados no Microsoft Excel, sendo realizada posteriormente a análise estatística de frequência e descrição dos resultados. Para correlacionar as variáveis avaliadas, utilizou-se o coeficiente de correlação de Spearman (rs) (SAMPAIO, 2007). A análise foi feita considerandoo nível de significância de $5 \%$.

\section{Resultados e Discussão}

A protrusão da glândula da terceira pálpebra foi diagnosticada por meio da visualização da alteração no exame oftálmico (Figura 1), sendo observado em 71 cães atendidos no período avaliado, correspondendo a $2,43 \%$ da casuística de cães no setor. A porcentagem de cães acometidos por esta alteração neste trabalho foi menor do que a observada na literatura (PEIXOTO; GALERA, 2012). Nenhum dos gatos atendidos pelo setor apresentou esta enfermidade, confirmando a baixa ocorrência nesta espécie (SLATTER, 2005).

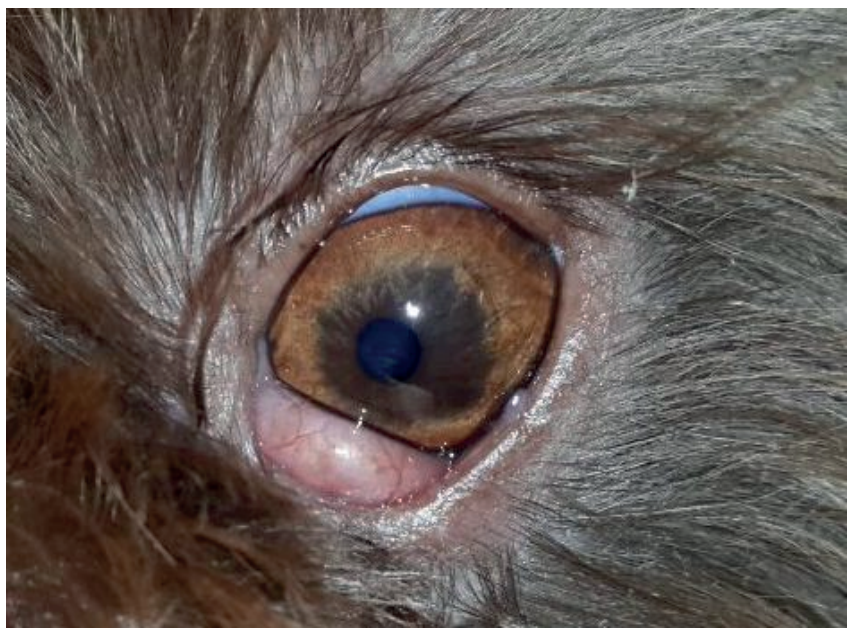

Figura 1: Cão diagnosticado com protrusão da glândula da terceira pálpebra, sendo visível a glândula exposta no canto medial do olho (Fonte: Serviço de Oftalmologia, FMVZ, Unesp-Botucatu).

Em algumas raças, suspeita-se que a protrusão da glândula da terceira pálpebra deva ser um defeito anatômico hereditário (WOUK; SOUZA; FARIAS, 2009), manifestando-se principalmente em animais com idade inferior a dois anos (HERRERA, 2008; PEIXOTO; GALERA, 2012). Neste estudo, $58 \%$ casos foram observados em filhotes (41/71), $32 \%(23 / 71)$ em adultos e $10 \%$ (7/71) em idosos, sendo que a idade dos animais variou de dois meses a 14 anos. Devido ao fato de parte dos proprietários encaminharem os animais para a avaliação tardiamente, justifica-se o atendimento desta alteração em animais idosos, apesar da afecção ter começado quando eram ainda jovens.

A maioria dos animais $(62 \%, 44 / 71)$ apresentou acometimento unilateral dos olhos, sendo que, do total, $27 \%$ (19/71)exibiram protrusãoda glândula da terceira pálpebra no olho direito e $35 \%(25 / 71)$ no olho esquerdo. O restan- 
te, $38 \%$ (27/71), dos cães apresentou a alteração bilateral. Segundo a literatura, a porcentagem média de animais com protrusão unilateral variou de 64 a 81\% (GALLEGO-MEJÍA et al., 2010; DEHGHAN et al., 2012; MAZZUCCHELLI et al., 2012; PEIXOTO; GALERA, 2012; SCALZILLI; BAUMWORCEL; SOARES, 2014), estando a porcentagem deste trabalho similar aos descritos anteriormente.

Cinquenta e cinco por cento (39/71) dos cães eram fêmeas e 45\% (32/71) machos, observando-se pequena diferença entre os sexos. Além disso, não houve correlação significativa entre este fator ea protrusão unilateral ou bilateral da glândula ( $\mathrm{rs}=0,18 ; \mathrm{p}=0,122)$ ou ao acometimento do olho direito ou esquerdo ( $\mathrm{rs}=-0,142 ; \mathrm{p}=0,234)$, indicando uma baixa influência do gênero na protrusão da glândula da terceira pálpebra. Apesar da discreta diferença de porcentagem, a maior frequência de fêmeas também foi relatada em trabalhos anteriores (GALLEGO-MEJÍA et al., 2010; PEIXOTO; GALERA, 2012; SCALZILLI; BAUMWORCEL; SOARES, 2014).

As raças mais acometidas pela protrusão da glândula da terceira pálpebra foram Lhasa Apso $(24 \%, 17 / 71)$, SRD $(20 \%, 14 / 71)$ e Beagle $(14 \%, 10 / 71)$. Porém outras raças também foram acometidas em menor porcentagem, como Shih-Tzu (8\%, 6/71), Bulldog Inglês (7\%, 5/71), Maltês (3\%, 2/71), Pinscher (3\%, 2/71), Poodle (3\%, 2/71), Rottweiler $(3 \%, 2 / 71)$ e Teckel $(3 \%, 2 / 71)$. As raças Boston Terrier, Bulldog Francês, Cane Corso, Cocker Spaniel Americano, Labrador Retriever, Mastim Napolitano, Pitbull, Pug e Yorkshire Terrier apresentaram apenas um caso cada (Figura 2). As raças observadas em maior frequência neste estudo foram semelhantes às já descritas na literatura (DEHGHAN et al., 2012; MAZZUCCHELLI et al., 2012; PEIXOTO; GALERA, 2012; SCALZILLI; BAUMWORCEL; SOARES, 2014). Entretanto, não foi observada correlação significativa entre a raça e a lateralidade da afecção ( $r s=-0,20 ; p=0,08$ ), não havendo, dessa forma, maior tendência à protrusão unilateral ou bilateral de acordo com as raças.

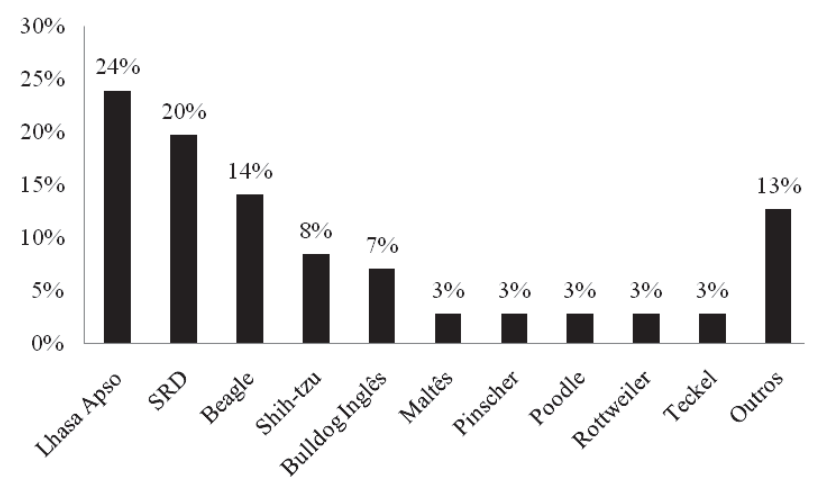

Figura 2: Principais raças acometidas pela protrusão da glândula da terceira pálpebra em cães atendidos no período de 2009 a 2013, pelo Hospital Veterinário da Faculdade de Medicina Veterinária e Zootecnia (UNESP), Campus Botucatu ( $\mathrm{n}=71$ ).

\section{Conclusão}

Conclui-se que a incidência de protrusão da glândula da terceira pálpebra é baixa em cães e rara em gatos e que não há influência sexual e racial quanto à lateralidade de apresentação clínica desta afecção.

\section{Referências}

CABRAL V.P. et al. Third eyelid gland protrusion in dogs. An experimental model proposal. Ciência Rural, v. 38, n.7, p. 1920-1924, 2008.

CHAHORY, S. et al. Three cases of prolapse of the nictitans gland in cats. Veterinary Ophthalmology,v. 7, n. 6, p. 417419, 2004.

DEHGHAN, M. M. et al. Clinical evaluation of the pocket technique for replacement of prolapsed gland of the third eyelid in dogs. Turkish Journal of Veterinary \& Animal Sciences,v. 36, n. 4, p. 352-356, 2012.

DUGAN S. J. et al. Clinical and histologic evaluation of the prolapsed third eyelid gland in dogs. Journal of the American Veterinary Medical Association, v. 201, n. 12, p. 1861-1867, 1992.

GALLEGO-MEJÍA, M. et al. Prolapso de glándula del tercer párpado en caninos atendidos

enel Hospital Veterinario de la Universidad de Caldas. Veterinaria y Zootecnia, v. 4, n. 1, p. 36-41, 2010.

HENDRIX, D. V. H. Canine conjunctiva and nictitating membrane. In: GELLAT, K.N. Veterinary Ophthalmology. Iowa: Blackwell publishing, 2007. p.662-689.

HERRERA, D. H. Enfermidadespalpebrais.In: Oftalmologia Clínica em Animais de Companhia. São Paulo: MedVet, 2008. p.89-110.

MAZZUCCHELLI, S. et al. Retrospective study of 155 cases of prolapse of the nictitating membrane gland in dogs. Veterinary Record, v. 170, n. 17, p.443, 2012.

PEIXOTO, R. V. R.; GALERA, P. D. Avaliação de 67 casos de protrusão da glândula da terceira pálpebra em cães (2005-2010). Arquivo Brasileiro de Medicina Veterinária e Zootecnia, v. 64, n. 5, p. 1151-1155, 2012.

SAMPAIO, I. V. B Testes estatísticos não paramétricos. In: $\quad$ Estatística aplicada à experimentação animal. 3. ed. Belo Horizonte: Fundação de Ensino e Pesquisa em Medicina Veterinária e Zootecnia, 2007. p.207-223.

SCALZILLI, B.; BAUMWORCEL, N.; SOARES, M. Estudo retrospectivo de cães diagnosticados com "cherry eye" (2003-2011). In: ANCLIVEPA, 35., 2014, Belo Horizonte. Anais... Belo Horizonte:CBA, 2014. p.788-790.

SCHOOFS, S. H. Prolapse of the gland of the third eyelid in a cat: A case report and literature review. Journal of the American Animal Hospital Association, v. 35, n. 3, p. 240-242, 1999.

SLATTER, D. Terceira Pálpebra. In: Fundamentos de Oftalmologia Veterinária. 3.ed. São Paulo: Roca, 2005. p. $247-257$. 
WOUK, A. F. P. F.; SOUZA, A. L. G.; FARIAS, M.

R. Afecções dos anexos oftálmicos. In: LAUS, J. L.

Oftalmologia clínica e cirúrgica em cães e gatos. São

Paulo: Roca, 2009. p.33-68.

Recebido em: 27/05/2014

Aceito em: 17/12/2014 